\title{
The Field of Charged, Spinning, Magnetic Particles
}

\author{
W. B. Bonnor and J. P. Ward \\ Queen Elizabeth College, Mathematics Department Atkins Building, London, England
}

Received July 25, 1972

\begin{abstract}
Stationary solutions of the Einstein-Maxwell equations have recently been given corresponding to charged, spinning magnetic matter. In this paper a solution for two particles of such matter is given. In general it contains a singularity between the particles.
\end{abstract}

\section{$\S 1$. Introduction}

Perjés [1] and Israel and Wilson [2] have recently given independently a new class of stationary solutions of the Einstein-Maxwell equations. These solutions describe the exterior field of massive, charged, magnetised, spinning particles. The electric charges and masses of the particles are such that the electric and gravitational forces between any two of them balance, and in this respect the solutions resemble those of Papapetrou [3] and Majumdar [4]. However, the particles also possess magnetism and spin, and in relativistic units the magnetic moment is equal to the angular momentum. In fact, every particle satisfies, in relativistic units $(c=1, G=1)$

$$
m=|e|, \quad \boldsymbol{h}= \pm \boldsymbol{\mu}
$$

$m, e, \boldsymbol{\mu}$ and $\boldsymbol{h}$ being the mass, charge, three dimensional magnetic moment and angular momentum.

In units of customary dimensions (1.1) become

$$
m \sqrt{G}=|e| \quad(a), \quad c^{-1} \sqrt{G} \boldsymbol{h}= \pm \boldsymbol{\mu} \quad \text { (b) }
$$

and objects with these parameters are physically quite plausible. For instance (a) is satisfied by a sphere of neutral hydrogen in which one atom in about $10^{18}$ has lost its electron; $|\boldsymbol{h}| /|\boldsymbol{\mu}|$ given by (b) is only about three orders of magnitude greater than the corresponding ratio for the Earth. Though objects satisfying both (a) and (b) together, even approximately, must be somewhat rare, it is clear that the matter described by the solutions is physically possible. It is therefore worth investigating them to see what insight they give into general relativity.

The solution for the simplest particle satisfying (1.1) was given by Perjés [1], and we propose to call it a Perjeon. If $\boldsymbol{h}=\boldsymbol{\mu}=0$ the particle 
will be called a Papapetron. (In fact, a Papapetron is a NordströmJefferies particle with $|e|=m$.)

The field equations used in this work are

$$
\begin{gathered}
R_{\mu v}=-8 \pi E_{\mu \nu}, \\
4 \pi E_{v}^{\mu}=-F^{\mu \alpha} F_{v \alpha}+\frac{1}{4} \delta_{v}^{\mu} F^{\alpha \beta} F_{\alpha \beta}, \\
F_{\mu v ; \sigma}+F_{v \sigma ; \mu}+F_{\sigma \mu ; v}=0, \\
F_{; v}^{\mu v}=J^{\mu}=0,
\end{gathered}
$$

where $E_{v}^{\mu}$ is the electromagnetic energy tensor, $F_{\mu v}$ the electromagnetic field tensor, and $J^{\mu}$ the four-current which vanishes because we consider only the exterior field. A semi colon denotes covariant differentiation with respect to the metric of space-time.

In this paper we obtain $(\S 2)$ the explicit exact solution for two Perjeons with spins (and magnetic moments) aligned parallel or antiparallel to their vector separation. Somewhat surprisingly, they cannot in general coexist in equilibrium, and there is in the solution a singularity between the particles. In this respect they are unlike Papapetrons. In $\S 3$ we attempt to give some physical meaning to the singularity, but are only partially successful. The paper ends with a Conclusion (\$4), and there is an Appendix.

\section{§ 2. The Solution for Two Perjeons}

We give first the general class of solutions already referred to $[1,2]$, in a somewhat modified notation. Latin indices run from 1 to 3 , and Greek from 1 to 4 . All functions are independent of $x^{4}$. A comma means partial differentiation and a stroke $\mid$ means covariant differentiation with respect to the metric $\gamma_{m n}$ to be defined now. The metric is

$$
d s^{2}=-f^{-1} \gamma_{m n} d x^{m} d x^{n}+f\left(\omega_{m} d x^{m}+d x^{4}\right)^{2},
$$

the three dimensional positive definite metric $\gamma_{m n}$ having zero Ricci Tensor; the electromagnetic field is given in terms of two scalar potentials:

$$
F_{4 n}=\phi_{, n}, \quad F^{a b}=\eta^{a b m} f \psi_{, m}
$$

$\eta^{a b m}$ being the Levi Civita symbol formed from the metric $\gamma_{m n}$. The entire solution is generated by two functions $L, M$, harmonic with respect to $\gamma_{m n}$ :

through the equations

$$
\gamma^{m n} L_{\mid m n}=0, \quad \gamma^{m n} M_{\mid m n}=0,
$$

$$
\begin{gathered}
\omega_{a, b}-\omega_{b, a}=+8\left(M L_{, t}-L M_{, t}\right) \eta_{a b m} \gamma^{m t}, \\
\phi=\frac{-\frac{1}{2} L \varepsilon}{L^{2}+M^{2}}, \quad \psi=\frac{\frac{1}{2} M \varepsilon}{L^{2}+M^{2}},
\end{gathered}
$$

where $\varepsilon= \pm 1$. 
Perjés gave the solution for a single Perjeon at the origin, namely $d s^{2}=-f^{-1}\left[d x^{2}+d y^{2}+d z^{2}\right]+f\left[d t+(y d x-x d y)\left(\frac{2 \mu}{R^{3}}+\frac{\mu m}{R^{4}}\right)\right]^{2}$, where $R=+\left(x^{2}+y^{2}+z^{2}\right)^{\frac{1}{2}}$, and $L, M$ are given by

$$
2 L=1+\frac{m}{R}, \quad 2 M=\frac{\mu z}{R^{3}} .
$$

The asymptotic values for the potentials of gravity, rotation, electricity and magnetism are respectively

$$
\begin{aligned}
& g_{44} \sim 1-\frac{2 m}{R} \\
& \frac{1}{2} y^{-1} g_{14}=-\frac{1}{2} x^{-1} g_{24}=\frac{\mu}{R^{3}} \\
& \phi \sim \frac{\varepsilon m}{R}+\mathrm{const} \\
& \psi \sim \frac{\varepsilon \mu z}{R^{3}}
\end{aligned}
$$

showing that the Perjeon has mass $m$, angular momentum $-\mu$, charge $\varepsilon m$ and magnetic moment $\varepsilon \mu$. Without fitting an interior solution to (2.9) one cannot say whether the magnetism can come from rotating charge or whether a permanent magnet is also necessary. However, if one considers the non-relativistic magnetostatic field of a rigidly rotating uniform and uniformly charged sphere one finds (in Gaussian units) [5]

$$
\frac{\mu}{h} \frac{m}{e}=\frac{1}{2 c}
$$

which is of the same order as in (1.2). Hence a configuration somewhat similar to this might produce the exterior field of a Perjeon without the need of permanent magnets.

A solution for two Perjeons at $A(0,0, a)$ and $B(0,0,-a)(a>0)$ is obtained by taking

$$
2 L=1+\frac{m_{1}}{r_{1}}+\frac{m_{2}}{r_{2}}, \quad 2 M=\frac{\mu_{1}(z-a)}{r_{1}{ }^{3}}+\frac{\mu_{2}(z+a)}{r_{2}{ }^{3}}
$$

where suffices 1 and 2 refer to the two particles, and

$$
r_{1}{ }^{2}=x^{2}+y^{2}+(z-a)^{2}, \quad r_{2}{ }^{2}=x^{2}+y^{2}+(z+a)^{2}
$$

$(x, y, z, t)$ being a general point in space-time. $L$ and $M$ satisfy (2.3) except at $r_{1}=0$ and $r_{2}=0, f$ is given by (2.4) and the electric and magnetic potentials by (2.6). An interaction term, absent in the case of two Papa- 
petrons, is given by $\omega$ in (2.5). The solution of this equation requires some calculation, and is undetermined up to the gradient of a scalar. The solution we wish to study, given in a common three dimensional vector notation is

$$
\begin{aligned}
\boldsymbol{\omega}= & (y \hat{\boldsymbol{i}}-x \hat{\boldsymbol{j}})\left\{\frac{\mu_{1}}{r_{1}{ }^{3}}\left(2+\frac{m_{1}}{r_{1}}\right)+\frac{\mu_{2}}{r_{2}{ }^{3}}\left(2+\frac{m_{2}}{r_{2}}\right)\right. \\
& +\frac{m_{1} \mu_{2}}{\varrho^{2}}\left[\frac{r_{1}}{2 a^{2} r_{2}}+\frac{(z+a)\left(\varrho^{2}+z^{2}-a^{2}\right)}{a r_{1} r_{2}{ }^{3}}\right] \\
& \left.+\frac{m_{2} \mu_{1}}{\varrho^{2}}\left[\frac{r_{2}}{2 a^{2} r_{1}}-\frac{(z-a)\left(\varrho^{2}+z^{2}-a^{2}\right)}{a r_{2} r_{1}{ }^{3}}\right]+\frac{K}{\varrho^{2}}\right\},
\end{aligned}
$$

where $K$ is an arbitrary constant and $\varrho^{2}=x^{2}+y^{2}$. Whatever the choice of $K$, this is singular somewhere on the rotation axis unless

$$
m_{1} \mu_{2}+m_{2} \mu_{1}=0 \text {. }
$$

If this condition is not fulfilled, then by a suitable choice of $K, \omega$ can be made non-singular on the axis either for $|z|>a$ or for $|z|<a$, but not both. We shall choose

$$
K=-\left(m_{1} \mu_{2}+m_{2} \mu_{1}\right) / 2 a^{2}
$$

which makes $\omega$ non-singular for $|z|>a$, but singular for $|z|<a$ unless (2.14) is satisfied.

For convenient reference we collect together the formulae which make up the complete solution for two Perjeons on the z-axis with spins and magnetic moments also parallel to the z-axis ${ }^{1}$

$$
\begin{aligned}
d s^{2}= & -4\left(L^{2}+M^{2}\right)\left(d x^{2}+d y^{2}+d z\right)+\frac{1}{4}\left(L^{2}+M^{2}\right)^{-1}(\omega \cdot d \boldsymbol{x}+d t)^{2} \\
2 L= & 1+\frac{m_{1}}{r_{1}}+\frac{m_{2}}{r_{2}}, \quad 2 M=\frac{\mu_{1}(z-a)}{r_{1}{ }^{3}}+\frac{\mu_{2}(z+a)}{r_{2}{ }^{3}}, \\
\phi= & \frac{-\frac{1}{2} \varepsilon L}{L^{2}+M^{2}}, \quad \psi=\frac{\frac{1}{2} \varepsilon M}{L^{2}+M^{2}}, \\
\omega= & (y \hat{i}-x \hat{j})\left\{\frac{\mu_{1}}{r_{1}^{3}}\left(2+\frac{m_{1}}{r_{1}}\right)+\frac{\mu_{2}}{r_{2}{ }^{3}}\left(2+\frac{m_{2}}{r_{2}}\right)\right. \\
& +\frac{m_{1} \mu_{2}}{\varrho^{2}}\left[-\frac{1}{2 a^{2}}+\frac{r_{1}}{2 a^{2} r_{2}}+\frac{(z+a)\left(\varrho^{2}+z^{2}-a^{2}\right)}{a r_{1} r_{2}{ }^{3}}\right] \\
& \left.+\frac{m_{2} \mu_{1}}{\varrho^{2}}\left[-\frac{1}{2 a^{2}}+\frac{r_{2}}{2 a^{2} r_{1}}-\frac{(z-a)\left(\varrho^{2}+z^{2}-a^{2}\right)}{a r_{2} r_{1}{ }^{3}}\right]\right\} .
\end{aligned}
$$

1 The precise connection between directions of spin and magnetic moment is governed by $\varepsilon$ in (2.6). For example, if the spins are both parallel to $\hat{k}$ (the positive $z$-direction), both magnetic moments must be parallel to $\hat{k}$, or both parallel to $-\hat{k}$. 
Where $d \boldsymbol{x}=(d x, d y, d z)$. The electromagnetic field is given by (2.2). This solution is valid in $-\infty<x, y, z, t<\infty$ except for the line (in 3 -space).

$$
|z| \leqq a, \quad x=y=0,
$$

unless (2.14) is satisfied in which case it is valid everywhere except at the two spatial points $(0,0, \pm a)$.

\section{§ 3. Physical Interpretation}

If one retains only terms linear in the four parameters $m_{1}, m_{2}, \mu_{1}, \mu_{2}$, and examines the asymptotic forms of metric and potentials, one sees by reference to (2.9) that one has at large distance the field of two Perjeons. The main interest of our solution (2.16) centres on the singularity (2.17), and we freely admit that we do not fully understand its physical meaning.

If $\mu_{1}=\mu_{2}=0$ the singularity disappears: indeed in this case we have two Papapetrons which, as is known, can coexist in equilibrium. The thought therefore arises that if $\mu_{1} \neq 0, \mu_{2} \neq 0$ the Perjeons are not in equilibrium unless (2.14) is satisfied, and the singularity represents a stress holding them in position. A somewhat similar situation arises with two mass points, for which the vacuum Einstein equations admit a static solution containing a stress singularity [6].

To pursue this hypothesis we could examine the behaviour of a Perjeon test particle at $A(0,0, a)$ in the field of a Perjeon at $B(0,0,-a)$. A fully-fledged Perjeon test particle is hard to treat because it is a charged, spinning, magnetised test particle, and thus has three interactions (electricity, spin and magnetism) which make it depart from geodesic motion. We can simplify the situation without losing its most interesting characteristic (the singularity (2.17)) by taking a test Papapetron instead of a test Perjeon, i.e. by putting $\mu_{1}=0$, and regarding $m_{1}$ as so small that its effect on $B$ is negligible. Note that from (2.14), if $\mu_{1}=0$ the singularity will be present provided $m_{1} \neq 0, \mu_{2} \neq 0$.

Let $e_{1}$ be the charge of the test Papapetron $A$ (numerically equal to $m_{1}$ ). The equation of motion of $A$ is

$$
\frac{d^{2} x^{\alpha}}{d s^{2}}+\Gamma_{\beta \gamma}^{\alpha} \frac{d x^{\beta}}{d s} \frac{d x^{\gamma}}{d s}=\frac{e_{1}}{m_{1}} F_{\beta}{ }^{\alpha} \frac{d x^{\beta}}{d s},
$$

and supposing it to be instantaneously at rest, we have

$$
\frac{d x^{\alpha}}{d s}=\left(0,0,0, f^{-\frac{1}{2}}\right)
$$

calculated at $x=y=0, z=a$. The remaining quantities in (3.1) are also to be calculated at $x=y=0, z=a$, and assuming that the self-field of $A$ 
must be ignored, we put $m_{1}=\mu_{1}=0$ in making the calculations using (2.16). We wish to find whether $A$ remains in equilibrium, that is whether $d^{2} x^{\alpha} / d s^{2}=0$.

The calculations are given in the Appendix. The important member of (3.1) is $\alpha=3$, and here the result is complicated (see A.3) and (A.4)), but essentially is that unless $\mu_{2}=0$ (i.e. unless $B$ as well as $A$ is a Papapetron) $d^{2} z / d s^{2} \neq 0$ and so the test particle $A$ is not in equilibrium in the field of $B$. In fact

$$
m_{1} \frac{d^{2} z}{d s^{2}}=\frac{m_{1} \mu_{2}^{2}}{16 a^{5}}-\frac{19}{128} \frac{m_{1} m_{2} \mu_{2}^{2}}{a^{6}}+\cdots
$$

and if $A$ is to remain at rest this must be balanced by a force which presumably is supplied in some way by the singularity.

However, it is not clear how the singularity in $\omega$ provides the force necessary to hold the particles in position. Its coefficient $m_{1} \mu_{2}$ is not of the right order to balance even the lowest term of the expression (3.3). Moreover, its appearance in $g_{14}$ and $g_{24}$ suggests a source of angular momentum, though not a simple monopole source of this.

The singularity seems to fulfil another function, connected with the asymptotic gravitational field. Expanding $\omega$ in (2.16) in powers of $R^{-1}=\left(x^{2}+y^{2}+z^{2}\right)^{-\frac{1}{2}}$ we find

$\boldsymbol{\omega}=(y \hat{\boldsymbol{i}}-x \hat{\boldsymbol{j}})\left\{\frac{2\left(\mu_{1}+\mu_{2}\right)}{R^{3}}+\frac{\mu_{1} m_{1}+\mu_{2} m_{2}}{R^{4}}+\frac{m_{1} \mu_{2}+m_{2} \mu_{1}}{R^{4}}+0(a)\right\}$,

the term $R^{-4}\left(m_{1} \mu_{2}+m_{2} \mu_{1}\right)$ coming from the singular parts of $\omega$, namely those containing the square brackets in (2.16). (3.4) can be written, ignoring terms of order $a$,

$$
\omega=(y \hat{i}-x \hat{j})\left\{\frac{2 \mu}{R^{3}}+\frac{m \mu}{R^{4}}\right\}
$$

where $m=m_{1}+m_{2}, \mu=\mu_{1}+\mu_{2}$, which from (2.7) is the correct form for a single Perjeon of parameters $m$ and $\mu$. Thus the singular terms in (2.16) are necessary for the system of two Perjeons to have the correct asymptotic form. The only circumstance in which the singularity can be dispensed with is if

which reduces to (2.14).

$$
m \mu=m_{1} \mu_{1}+m_{2} \mu_{2},
$$

The singularity discussed here should be distinguished from those considered by Hartle and Hawking [7]. The latter also use the solution (2.1)-(2.6), but choose both $L$ and $M$ to be of order $R^{-1}$. They so obtain a charged NUT solution, or superposition of them. Since the singularity structure of a single, uncharged, NUT solution is known to be rather abstruse $[8,9]$ the work of Hartle and Hawking seems even harder to interpret than our own. 


\section{§ 4. Conclusion}

Two Perjeons, unlike two Papapetrons, are not in equilibrium unless (2.14) is satisfied, and this fact must be connected with the singularity in $\omega$ on the line between them. However, it is not clear what physical object this singularity represents. The singularity also serves to make the twoPerjeon solution satisfy appropriate conditions at spatial infinity.

\section{Appendix}

The Force on a Test Papapetron $A$ in the field of a Perjeon $B$

We use metric (2.16) with $\mu_{1}=m_{1}=0$, and (3.1) in the form

$$
\frac{d^{2} x^{\alpha}}{d s^{2}}=\lambda F_{\beta}{ }^{\alpha} \frac{d x^{\beta}}{d s}-\Gamma_{\beta \gamma}^{\alpha} \frac{d x^{\beta}}{d s} \frac{d x^{\gamma}}{d s}
$$

where $\lambda$ is the charge to mass ratio of $A$ (either + or -1 ). There is of course no contradiction in ignoring $m_{1}$ in (2.16) (which amounts to neglecting $A$ 's self field) and retaining it in (3.1) (where it is necessary, in the form of inertial mass, to make $A$ respond to $B$ 's field). Using (2.1), (2.2) and (2.16) we obtain, when $\alpha=3$

$$
\begin{aligned}
\frac{d^{2} z}{d s^{2}}= & \frac{1}{4} \lambda\left(L^{2}+M^{2}\right)^{-\frac{1}{2}} \frac{\partial}{\partial z}\left\{\frac{\varepsilon L}{L^{2}+M^{2}}\right\}-\frac{1}{8} \frac{\partial}{\partial z}\left(L^{2}+M^{2}\right)^{-1} \\
= & \frac{1}{4}\left(L^{2}+M^{2}\right)^{-5 / 2}\left\{L_{z}\left[L\left(L^{2}+M^{2}\right)^{\frac{1}{2}}+\lambda \varepsilon\left(M^{2}-L^{2}\right)\right]\right. \\
& \left.+M M_{z}\left[\left(L^{2}+M^{2}\right)^{\frac{1}{2}}-2 \lambda \varepsilon L\right]\right\}
\end{aligned}
$$

positive square roots being taken everywhere. We now insert for $L$ and $M$ the expressions in (2.16) with $m=\mu_{1}=0$, namely

$$
2 L=1+\frac{m_{2}}{r_{2}}, \quad 2 M=\frac{\mu_{2}(z+a)}{r_{2}^{3}},
$$

and putting $z=a, x=y=0$ after differentiation we obtain for the $z$-component of the acceleration of $A$

$$
\begin{aligned}
\left(\frac{d^{2} z}{d s^{2}}\right)_{A}= & {\left[P^{2}+Q^{2}\right]^{-5 / 2}\left\{\frac{m_{2}}{4 a^{2}}\left[\lambda \varepsilon\left(P^{2}-Q^{2}\right)-P\left(P^{2}+Q^{2}\right)^{\frac{1}{2}}\right]\right.} \\
& \left.+\frac{Q^{2}}{a}\left[2 \lambda \varepsilon P-\sqrt{P^{2}+Q^{2}}\right]\right\}
\end{aligned}
$$

where $P=1+\frac{m_{2}}{2 a}, Q=\frac{\mu_{2}}{4 a^{2}}$. If we put $\mu_{2}=0$ we obtain

$$
\frac{d^{2} z}{d s^{2}}=\frac{m_{2}}{4 a^{2} P^{3}}(\lambda \varepsilon-1),
$$


so that $d^{2} z / d s^{2}=0$ if $\lambda \varepsilon=1$. This is the case of Papapetrons with $e / m$ of the same sign for both $B$ and the test particle $A$, so there is equilibrium.

If $\mu_{2} \neq 0$ we expand (A.3) in powers of the parameters $m_{2}, \mu_{2}$, and put $\lambda \varepsilon=1$ corresponding to a test Papapetron; then

$$
\frac{d^{2} z}{d s^{2}}=\frac{\mu_{2}^{2}}{16 a^{5}}-\frac{19}{128} \frac{m_{2} \mu_{2}{ }^{2}}{a^{6}}+0\left(m_{2}{ }^{2} \mu_{2}{ }^{2} ; \mu_{2}{ }^{4}\right),
$$

giving the acceleration which must be balanced by a force if $A$ is to remain at rest.

\section{References}

1. Perjés,Z.: Phys. Rev. Letters 27, 1668 (1971).

2. Israel, W., Wilson, G. A.: J. Math. Phys. 13, 865 (1972).

3. Papapetrou, A.: Proc. Roy. Irish Acad. 51, 191 (1947).

4. Majumdar, S. D.: Phys. Rev. 72, 390 (1947).

5. Panofsky, W.K.H., Phillips, M.: Classical Electricity and Magnetism, p. 131. Reading, Mass.: Addison-Wesley 1969.

6. Synge, J.L.: Relativity - The General Theory, p. 315. Amsterdam: North-Holland 1960.

7. Hartle, J. B., Hawking, S. W.: Commun. math. Phys. 26, 87 (1972).

8. Misner, C. W.: Contribution to lectures in applied mathematics, Vol. 8, p. 160. Am. Math. Soc. 1967.

9. Bonnor, W. B.: Proc. Cambridge Phil. Soc. 66, 145 (1969).

W. B. Bonnor

J. P. Ward

Queen Elizabeth College

Mathematics Department

Atkins Building

Campden Hill

London, W. 8., England 